\title{
Nutritional support in per operative period: the use of lipid solutions
}

\author{
F. Mandolfino*, M. Frascio, C. Stabilini, F. Lazzara, M. Imperatore, E. Gianetta \\ Dipartimento di Scienze Chirurgiche (DISC), Università di Genova, \\ Largo R. Benzi 10, 16132 Genova \\ *succlavia@yahoo.it
}

\begin{abstract}
Energy deficit is a common and serious problem in major surgical patients and is associated with increased rates of complications, length of stay, and mortality. Parenteral nutrition (PN), either alone or in combination with enteral nutrition, can improve nutrient delivery to this kind of patients. Lipids provide a key source of calories within PN formulations, preventing or correcting energy deficits and improving outcomes. In this article, we review the role of parenteral lipid emulsions (LEs) in the management of patients undergone to major surgical procedures and highlight important biologic activities associated with lipids. Soybean-oil-based LEs with high contents of polyunsaturated fatty acids (PUFA) were the first widely used formulations in the surgical setting. More recently developed parenteral LEs employ partial substitution of soybean oil with oils providing medium-chain triglycerides, omega-9 monounsaturated fatty acids or omega-3 PUFA. Many of these LEs have demonstrated reduced effects on oxidative stress, immune responses, and inflammation. Ongoing research using adequately designed and wellcontrolled studies that characterize the biological properties of LEs should assist clinicians in selecting LEs within the surgical setting.
\end{abstract}

\section{Introduction}

Artificial nutrition support refers to the administration of nutrient solutions by the enteral or parenteral route. It's an old concept: one of the first experiences was reported by a group of surgeons interested in the positive effects of longterm total parenteral nutrition in the management of major gastrointestinal surgery.

Per operative nutritional intervention is an integral component of preoperative preparation and postoperative therapeutic strategy in surgical patients with undernutrition or in patients at severe nutritional risk. The guidelines based on clinical trials highlight the risk groups with benefit of artificial nutrition [1].

The aim of nutritional treatment is to prevent malnutrition, or to correct nutritional depletion where it already exists, avoiding surgical complications and improving the clinical outcome of surgical patients. Indications to artificial support are mandatory and based on Clinical Guidelines for the Use of Parenteral and Enteral Nutrition in Adult and Paediatric Patients [2]. Enteral nutrition is mandatory if gut works. Tube feeding is required in patients with profound anorexia, swallowing disorders, and occasionally to fully utilise available intestinal function by overnight infusion. Where prolonged tube feeding is anticipated, for example 2-4 weeks, Percutaneous gastrostomy (PEG) tubes should be placed. The surgical placement of a percutaneous jejunostomy tube will provide a suitable route for nutrient administration in some patients who are undergoing upper alimentary surgery, in whom oral nutrition is not anticipated soon after the operation [3]. Parenteral nutrition should be reserved to patients whose gut can't be used or when intestinal function is inadequate or the intestine is not available.

Our experience refers to the composition of parenteral nutrition and particularly the use of lipids.

Role of parenteral lipid emulsion for surgical patients

Lipids, respect to carbohydrates, provide two major advantages for total parenteral nutrition. First, they provide essential fatty acids, thus avoiding essential fatty acid deficiency, and secondly, they are a useful energy source, providing $9 \mathrm{kcal} / \mathrm{g} .20$ to $30 \%$ of total calories are usually supplied as lipids [4].

Use of lipid emulsions is useful, in adult patients in case of hearth, kidney or respiratory failure.

The conventional lipid emulsions (soybean oil) are based on long-chain triglycerides (LCT) derived from soybean or safflower oil. They can be characterized as the first generation of emulsions.

The so called "Medium Chain Triglycerides (MCT)/LCT" lipid emulsions was developed in the 1980s. They present several advantages in comparison to LCT lipid emulsions, in particular providing more efficient fuel without interfere with pulmonary hemodynamics or gas exchange.

As an alternative to a physical mixture of MCT and LCT, interesterification of Medium Chain Fatty Acid (MCFA) and 
Long Chain Fatty Acid (LCFA) is possible to create mixed triglycerides, so called structured lipids. Randomized SL are synthesized by simply mixing MCT and LCT oils and heating the mixture in the presence of a catalyst.

The choice of parenteral LE should be based upon several considerations. It has been suggested that the ratio of linoleic acid (LA) to alpha-linolenic acid (ALA) is important because of competition between these fatty acid (FA) for a number of enzymes, thereby potentially influencing the production of eicosanoid and eicosanoid-like inflammatory mediators [5].

The stability of LEs, with respect to phase separation and presence of large globules, is also an important issue in clinical settings in which the final concentrations of lipid components and emulsifiers are of key importance. All LEs eventually become unstable when diluted above a certain threshold within the PN formulation.

In general, MCT/soybean oil and olive-oil-rich LEs have demonstrated greater stability when compared with soybean- oil- and safflower-oil-based LEs, although stability may vary between manufacturers $[5,6]$. The stability of MCT/soybean oil LEs may be due to the inclusion of shorter-chain lipids, which exhibit a lower free energy when dispersed in water, resulting in greater miscibility between phases and reduced physicochemical stress on emulsifying agents compared with LEs containing predominantly longer-chain triglycerides [7]. Olive-oil-rich LEs contain sodium oleate, which acts as an additional emulsifying agent and thereby augments stability [7]. In addition, the stability of PN formulations that contain LEs may be affected by interactions between FA and other commonly administered compounds, such as carnitine, heparin, and some vitamins $[8,9]$.

The biological effects associated with LEs are likely to benefit a majority of patients receiving parenteral LEs, including those on long-term total PN, but may have the greatest importance for patients under metabolic stress. Therefore, physicians need to consider several issues when selecting an LE as part of a PN regimen.

All currently available LEs provide sufficient $x-6$ and $x-3$ Essential Fatty Acid (EFA), with the exception of $100 \%$ fish oil LE, which should generally only be used as a pharmacological agent or as a supplement to other LEs $[10,11]$.Although there are conflicting views regarding the comparative utility of different LE formulations in surgical patients, there is growing consensus that LEs based entirely on soybean oil should be avoided in favour of emulsions in which the LA and ALA content is partially replaced by MCT, olive oil providing MUFA or fish oil providing eicosapentaenoic acid (EPA) and docosahexaenoic acid (DHA). This is particularly true for patients with highly proinflammatory states, such as surgical, trauma, burn, and septic patients [12].

\section{Conclusions}

Lipids are an important source of calories in artificial nutrition, and they have demonstrated a wide range of biological activities that may benefit a variety of patients receiving $\mathrm{PN}$, as well as those receiving $\mathrm{EN}$ with or without $\mathrm{PN}$ supplementation.

Newer LEs that utilize partial substitution of soybean oil with MCT, olive oil or fish oil either alone or in combination have demonstrated potential benefits in terms of reduced impacts on oxidative stress and differential effects on cell-mediated immunity and inflammation. These studies must be adequately designed and well controlled. Until then, the prescription of LEs should be based upon the limited clinical data available, the range of available LEs, cost implications, and an understanding of the potential biological effects of their components, bearing in mind the situation and therapeutic goals of the individual patient.

\section{References}

[1] Adolph M. 1999. Lipid emulsions in parenteral nutrition.Ann. Nutr. Metab., 43: 1-13.

[2] American Society for Parenteral and Enteral Nutrition Board of Directors 2009. Clinical guidelines for the Use of Parenteral and Enteral Nutrition in Adult and Pediatric Patients.

[3] Lewis S. J., Andersen H. K., Thomas S. 2009. Early enteral nutrition within $24 \mathrm{~h}$ of intestinal surgery versus later commencement of feeding: a systematic review and metaanalysis. J. Gastroint. Surg., 13(3): 569-575.

[4] Crook M.A. 2000. Lipid clearance and total parenteral nutrition: the importance of monitoring plasma lipids. Nutrition, 16(9): 774-775.

[5] Stanley J.C., Elsom R.L., Calder P.C., Griffin B.A., Harris W.S., Jebb S.A., Lovegrove J.A., Moore C.S., Riemersma R.A., Sanders T.A. 2007. UK Food Standards Agency Workshop Report: the effects of the dietary $n-6: n-3$ fatty acid ratio on cardiovascular health. Br.J. Nutr., 98: 1305-1310.

[6] Driscoll D.F. 2007. Globule-size distribution in injectable $20 \%$ lipid emulsions: Compliance with USP requirements.Am. J. Health Syst. Pharm., 64: 2032-2036.

[7] Driscoll D.F., Giampietro K., Wichelhaus D.P., Peterss H., Nehne J., Niemann W., Bistrian B.R. 2001. Physicochemical stability assessments of lipid emulsions of varying oil composition. Clin. Nutr., 20: 151-157.

[8] Berger M.M., Shenkin A. 2006.Vitamins and trace elements: practical aspects of supplementation. Nutrition, 22: 952-955

[9] Van Aerde T. 2004. In preterm infants, does the supplementation of carnitine to parenteral nutrition improve the following clinical outcomes: growth, lipid metabolism and apneic spells?: Part A: evidence-based answer and summary. Paediatr. Child Health, 9:571- 572.

[10] Wanten G.].A., Calder P.C. 2007. Immune modulation by parenteral lipid emulsions. Am. J. Clin. Nutr., 85: 1171-1184.

[11] McClave S.A., Martindale R.G.,Vanek V.W., McCarthy M., Roberts P., Taylor B., Ochoa J.B., Napolitano L., Cresci G. 2009. Guidelines for the provision and assessment of nutrition support therapy in the adult critically ill patient: Society of Critical Care Medicine (SCCM) and American Society for Parenteral and Enteral Nutrition (A.S.P.E.N.).

[12] Calder P.C. 2007. Immunonutrition in surgical and critically ill patients. Br. J. Nutr., 98(1): S133-S139. 


\title{
The use of a masticatory robot to analyze the shock absorption capacity of different restorative materials for implant prosthesis
}

\author{
M. Menini'*, E. Conserva', T. Tealdo', M. Bevilacqua', F. Pera², G. Ravera³, P. Pera' \\ ' Department of Fixed and Implant Prosthodontics, University of Genova, L. Rosanna Benzi 10, 16132 Genova, Italy \\ ${ }^{2}$ Department of Prosthodontics, University of Turin, V. Santena 7, 10126 Turin, Italy \\ ${ }^{3}$ Department of Health Sciences, Section of Biostatistics, University of Genova, V. A. Pastore I, 16132 Genova, Italy \\ *maria.menini@unige.it
}

KEYWORDS: dental materials; force transmission; implant; chewing simulator

\begin{abstract}
The aim of the present research was to measure in vitro the chewing load forces transmitted through crowns made of different prosthetic restorative materials onto the dental implant. A masticatory robot that is able to reproduce the mandibular movements and the forces exerted during mastication was used. The forces transmitted to the simulated periimplant bone during the robot mastication were analyzed, using different occlusal materials. Two-way analysis of variance (ANOVA) was used. The zirconia and the ceramic crowns transmitted significantly greater forces ( $p$-value $<0.000 \mathrm{l}$ ) than the other crowns tested. Dental materials with lower elastic modulus were better able to absorb shock from occlusal forces than more rigid materials.
\end{abstract}

\section{Introduction}

The occlusal load is a critical factor in reaching and maintaining osseointegration, and therefore affecting the long term survival of dental implants. The restorative material used in dental implants may be one of the factors affecting the forces transmitted to the periimplant bone [13]. The aim of the present study is to investigate in vitro the shock absorption capacity of dental restorative materials currently in use.

\section{Materials and Methods}

A masticatory robot was used, which is able to simulate human chewing in vitro, reproducing three-dimensionally mastication. The robot, which simulates an implant setup, has been described and validated in a previous paper [1] The materials tested were: zirconia (Procera), a glass ceramic (Empress 2), a gold alloy (Ney-Oro cb), two composites (Experience, Signum), an acrylic resin (Easytemp 2) (Tab. 1). Each crown was positioned on the implant abutment simulation of the robot and placed under 350 chewing cycles with the sample crown occluding with the chrome-cobalt steel upper arch (Fig. 1).

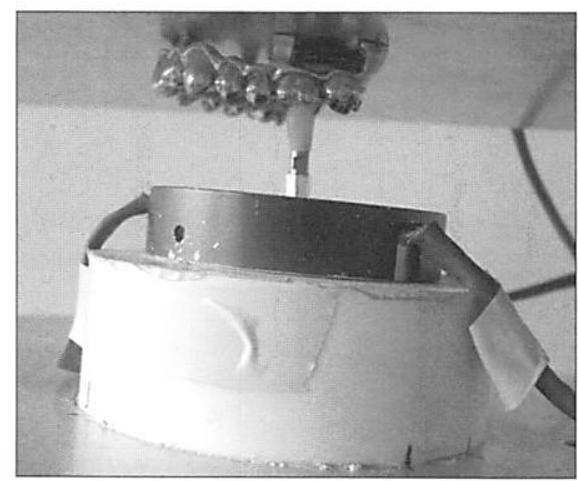

Figure I. Detail of the sensor-equipped masticatory robot

Vertical loads transmitted at the simulated bone-implant interface were recorded. The maximum values of the forces recorded for each masticatory cycle were highlighted and underwent statistical analysis with SPSS software (Version 13.0, SPSS Inc.). Two-way analysis of variance (ANOVA) was used to compare transmitted stresses between the different occlusal materials tested.Alpha was set at .05.

\section{Results}

The ANOVA test found a significant ratio between the variations and so Scheffe's post hoc test was applied: within the materials an internal comparison showed a significant result with $p$-value $<0.0001$ (Tab. 1).

The slope of the curve, representing the force transmitted at the periimplant level, showed that materials with greater elastic modulus have steeper peaks than the other materials, that is that the maximum force is reached more rapidly. 


\begin{tabular}{|c|c|c|c|c|c|c|}
\hline Materials testod & Manufacturer & $\begin{array}{l}\text { Elastic } \\
\text { Modulus } \\
\text { (MPa) }\end{array}$ & $\begin{array}{l}\text { Mean } \\
\text { (kg) }\end{array}$ & $\begin{array}{l}\text { Dev. } \\
\text { Std. }\end{array}$ & $\begin{array}{l}\text { Er. } \\
\text { std }\end{array}$ & $\%$ \\
\hline Procera Zirconia & Nobel Biocare & 210000 & 39.529 & 1.378 & 0.097 & - \\
\hline Empress 2 & Ivodar Vivaden & 96000 & 0.127 & 0.572 & 0.031 & -23.79 \\
\hline Ney-Orocb & Dentsply & 77000 & 20.761 & 1.207 & 0.070 & -47.48 \\
\hline & DEI(@italia & 130 & 91 & 1.085 & 8 & -55.75 \\
\hline Signum & Heraeus Kulzer & 3500 & 9.510 & 0.507 & 0.027 & -75.94 \\
\hline Easytemp 2 & DEI(x)italia & 2300 & 2.471 & 0.663 & 0.035 & -93.75 \\
\hline
\end{tabular}

Table 1. Comparison of mean maximum occlusal force $(\mathrm{kg})$.

\section{Discussion}

The use of different restorative materials significantly affected stress transmission at the simulated bone-implant interface. In fact the masticatory robot recorded values of forces transmitted significantly higher when using materials with higher elastic modulus with respect to other materials. Zirconia and ceramic crowns also showed steeper peaks of force than the other materials. This was considered another effect of the different elastic modulus of the materials tested. Acrylic resin was able to reduce the forces transmitted at the simulated bone-implant interface up to $-93.75 \%$ with respect to zirconia. It is the authors opinion that these findings should be taken into consideration in clinical decision making especially in the clinical situations (such as immediate loading protocols) and in particular unfavourable conditions (such as parafunctions) when implant loading needs to be limited.The use of an acrylic resin provisional prosthesis or a definitive composite resin prosthesis is essential in such situations to reduce the risk of overload in a critical moment for the obtainment or maintenance of osseointegration.

\section{References}

[1] Conserva E., Menini M., Tealdo T., Bevilacqua M., Pera F., Ravera G., Pera P. 2008. Robotic chewing simulator for dental materials testing on a sensor-equipped implant set up. Int. J. Prosthodont., 21:501-508.

[2] Conserva E., Menini M., Tealdo T., Bevilacqua M., Ravera G., Pera F., Pera P. 2009. The use of a masticatory robot to analyze the shock absorption capacity of different restorative materials for prosthetic implants: a preliminary report. Int. J. Prosthodont., 22:53-55.

[3] Skalak R. 1983. Biomechanical considerations in osseointegrated prostheses. J. Prosthet. Dent., 49:843-848. 